\title{
Convection in RR Lyrae Stars
}

\author{
R. F. Stellingwerf ${ }^{1}$, G. Bono ${ }^{2}$ \\ ${ }^{1}$ Los Alamos National Laboratory Los Alamos, NM, ${ }^{2}$ Trieste Observatory, Trieste, Italy
}

\begin{abstract}
Convection undoubtedly plays a strong role in defining the RR Lyrae instability strip, but its effects on amplitude, mode of pulsation, and light curve have always been problematical. One reason is simply that convective models are difficult to compute, and their accuracy is difficult to ascertain. We present results of a new convective survey of RR Lyrae stars that constitute the best models available at this time, with better boundary conditions, physics, zoning, and length of computational run than previous results.
\end{abstract}

\section{Introduction}

Attempts at inclusion of convection in pulsating star models date from the early work by Baker and Kippenhahn (1962). They used linear non-adiabatic models in which the convective effects were included in the static structure of the star, but the time dependence was not treated in detail in the pulsation model. Pulsational damping was found for cooler models, but the width of the instability strip as computed was too large, and the phase shifts of the cooler models did not match those of observed stars. This study set the tone of subsequent endeavors in the field of time-dependent convection in the sense that comparison with only a few observational parameters (width of strip, phase of light curve, amplitudes, etc.) comprise the only tests of the theory's validity. Christy (1966) showed that non-convective models seemed to match many of the properties of observed stars, except for the quenching at the red edge of the instability strip. Later work showed that nonconvective models tended toward amplitudes larger than observed, and showed difficulties with mode selection - particularly modeling the RRd mixed-mode stars. Convective models, although undoubtedly over-simplifications of reality, may be considered "successful" if some or all of these discrepancies are removed.

Convective theories may be classified according to several characteristics: 1) dimensionality (1D or 2D), 2) effect of the convection on the adjacent regions (either local or nonlocal), 3) amplitude (either linear or nonlinear), and 4) time dependence of the convection (either time-dependent or frozen). Unno (1967) and Baker and Gough (1979) tried linear, local, time-dependent theories, Cox, et al. (1966), and Wood (1979) tried nonlinear, local, time-dependent theories. These attempts all showed promise, but missed significant features of the observations. Deupree (1979) tried a 2D nonlinear theory that matched observations well in many respects. Stellingwerf (1982) derived a 1D nonlinear, nonlocal, time-dependent theory, which is somewhat easier to run and interpret than Deupree's 2-dimensional scheme, and which also seemed to match observation quite well.

This paper presents an overview of a new series of computations using this scheme. For details, see the presentation by Bono in this collection. Here we present a qualitative review of the results and their implications. 


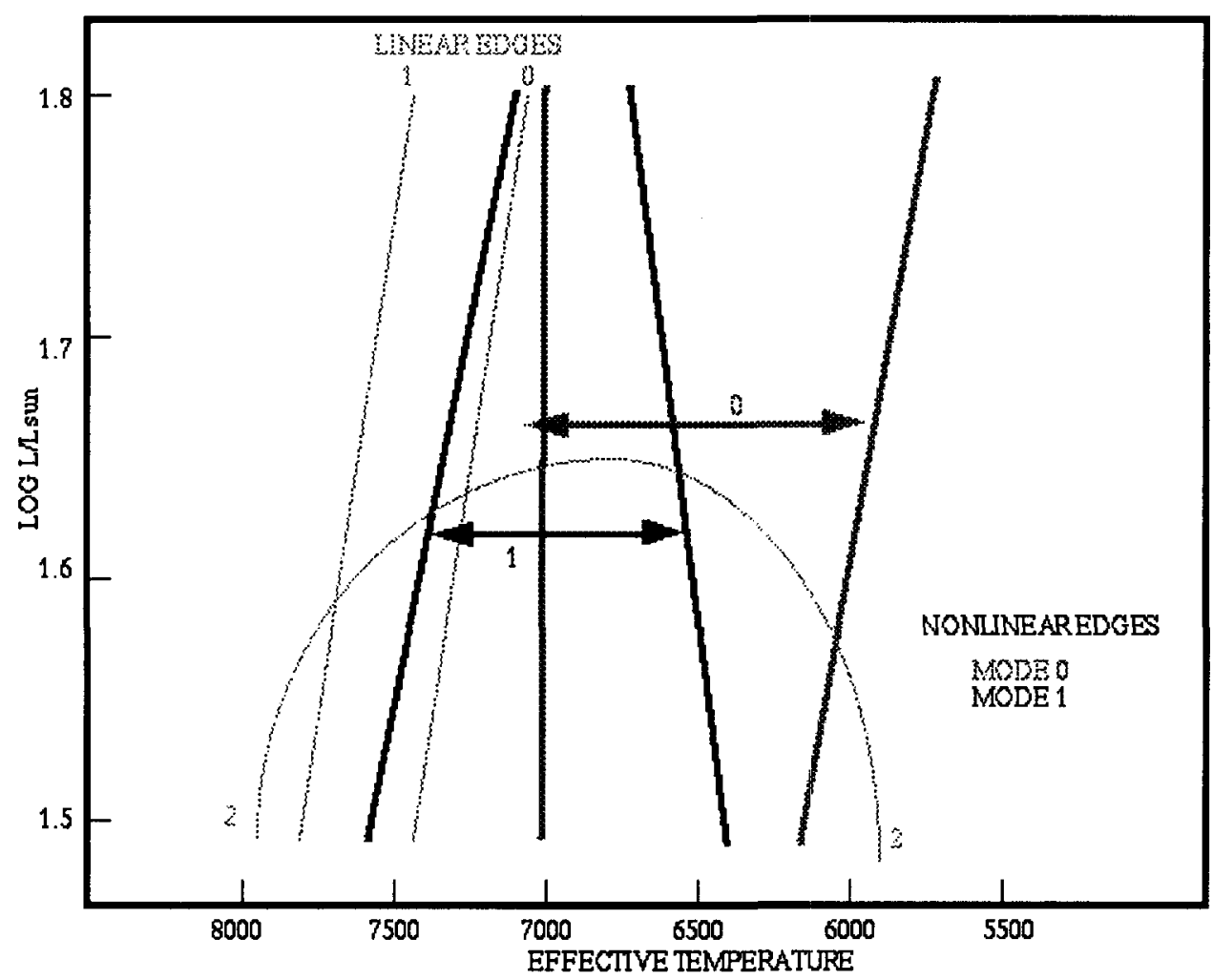

Figure 1: Location of the linear and nonlinear instability strips on the HR diagram.

\section{Instability Strip}

Figure 1 shows the location of the instability strip of the RR Lyrae stars with mass 0.65 and composition $\mathrm{X}=0.7, \mathrm{Z}=0.001$. Linear radiative models were computed for the fundamental (mode 0 ), first overtone (mode 1), and second overtone (mode 2 ) modes. The blue edges are shown above as grey lines. Only the second overtone is stabilized toward the red within the limits of this diagram; this is the normal result for non-convective models. These models have about 120 radial zones, which is fine enough to resolve all of the important features of the pulsation.

The models were then repeated using a nonlinear, convective code with the same physics, numerics, and parameters. Only fundamental and first overtone modes have been computed at this time. Models were computed at four different luminosities. The nonlinear models were integrated about 500 periods to determine their final preferred mode. The dark lines shown above show the final position of the nonlinear instability strips for the fundamental and first overtone modes. These lines are smooth approximations to the detailed results, but should be accurate within the uncertainties of the survey. The implications of these results will be discussed below.

Note that the blue edges are considerably shifted toward the red, and the slope of the fundamental blue edge is considerably changed from the linear results. This illustrates the changes in this survey from the "classical" linear, radiative results. These changes represent a combination of the effects of convection and nonlinearities, to be unraveled in the next section. Of course, another change is the appearance of the red edges at the observed locations. 


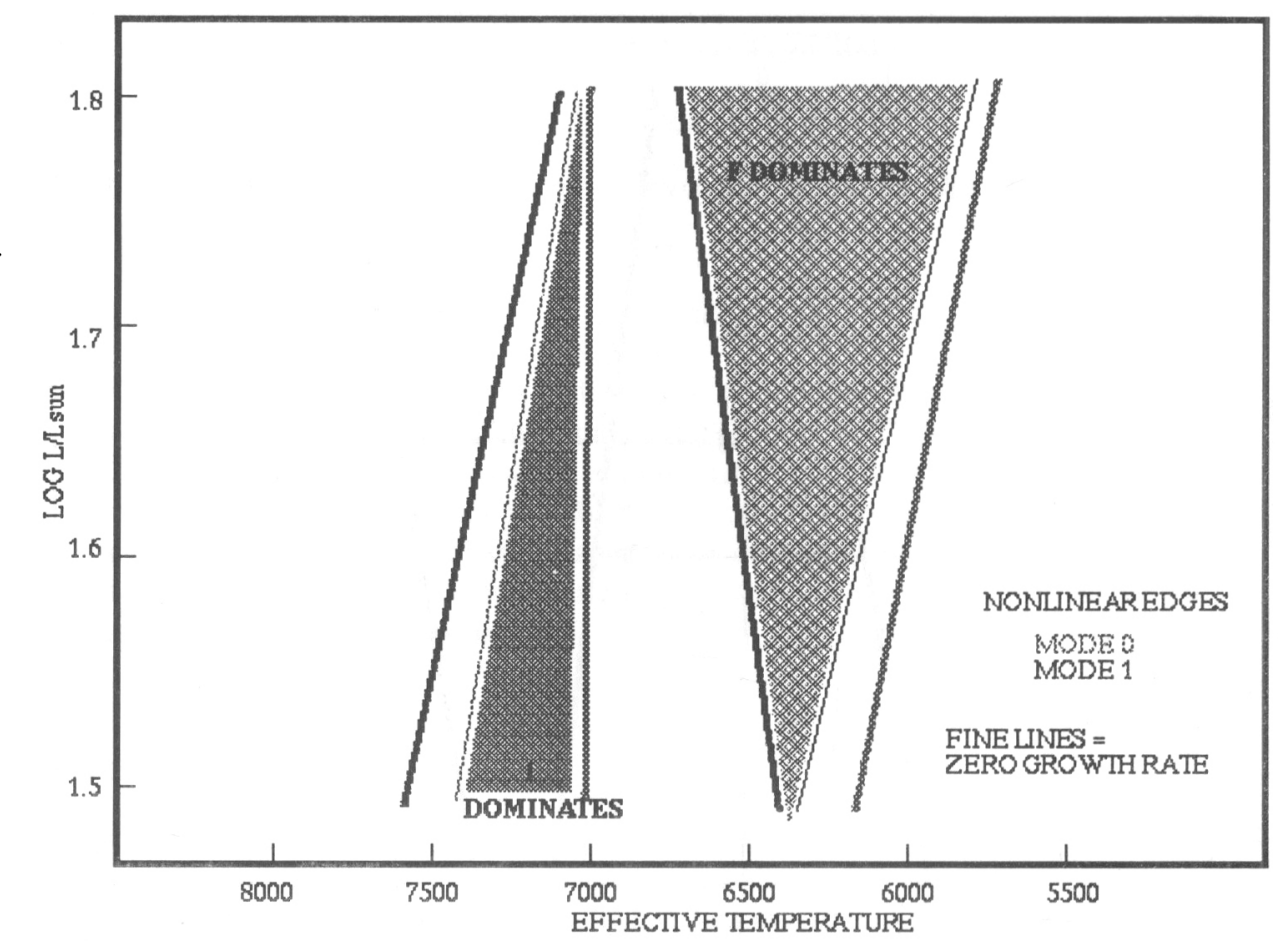

Figure 2: Nonlinear instability strip showing the locations of the "zero growth rate lines" - these are the linear / convective edges of the instability strip as derived from the nonlinear models.

\section{Mode Switching}

Another piece of information derived from the nonlinear models is the slope of the growth rate at early time during the integration. Since the integration is started at modest amplitude $(10 \mathrm{~km} / \mathrm{s}$ velocity), this gives an approximation to the linear, convective instability strip (this strip was not computed directly because of the difficulty of including the nonlocal effects in the linear analysis). In this way we can separate the effects of the convection from those of the nonlinearity. Figure 2 shows the edges obtained in this manner, as well as the nonlinear results shown in Figure 1. Only the blue fundamental "zero growth" line and the red overtone "zero growth" line are shown, since the other lines coincide with the nonlinear edges. In the cases shown the nonlinear strips begin relatively far from the lines of zero growth, indicating that models in these regions would begin pulsating in one mode, but switch to the other before attaining full amplitude. These "mode switching" regions are shown as shaded regions in Figure 2. The diagonal line at about $6500 \mathrm{~K}$ is often referred to as the "mode-switch" line. As seen from the figure, however, there are actually two mode switching lines, the usual line at $6500 \mathrm{~K}$, which is actually the overtone red edge for the nonlinear models (at this line redward evolving overtone pulsators will switch to the fundamental mode), and one at about $7000 \mathrm{~K}$, which is the fundamental nonlinear blue edge (at this line blueward fundamental pulsators will switch to the overtone). 


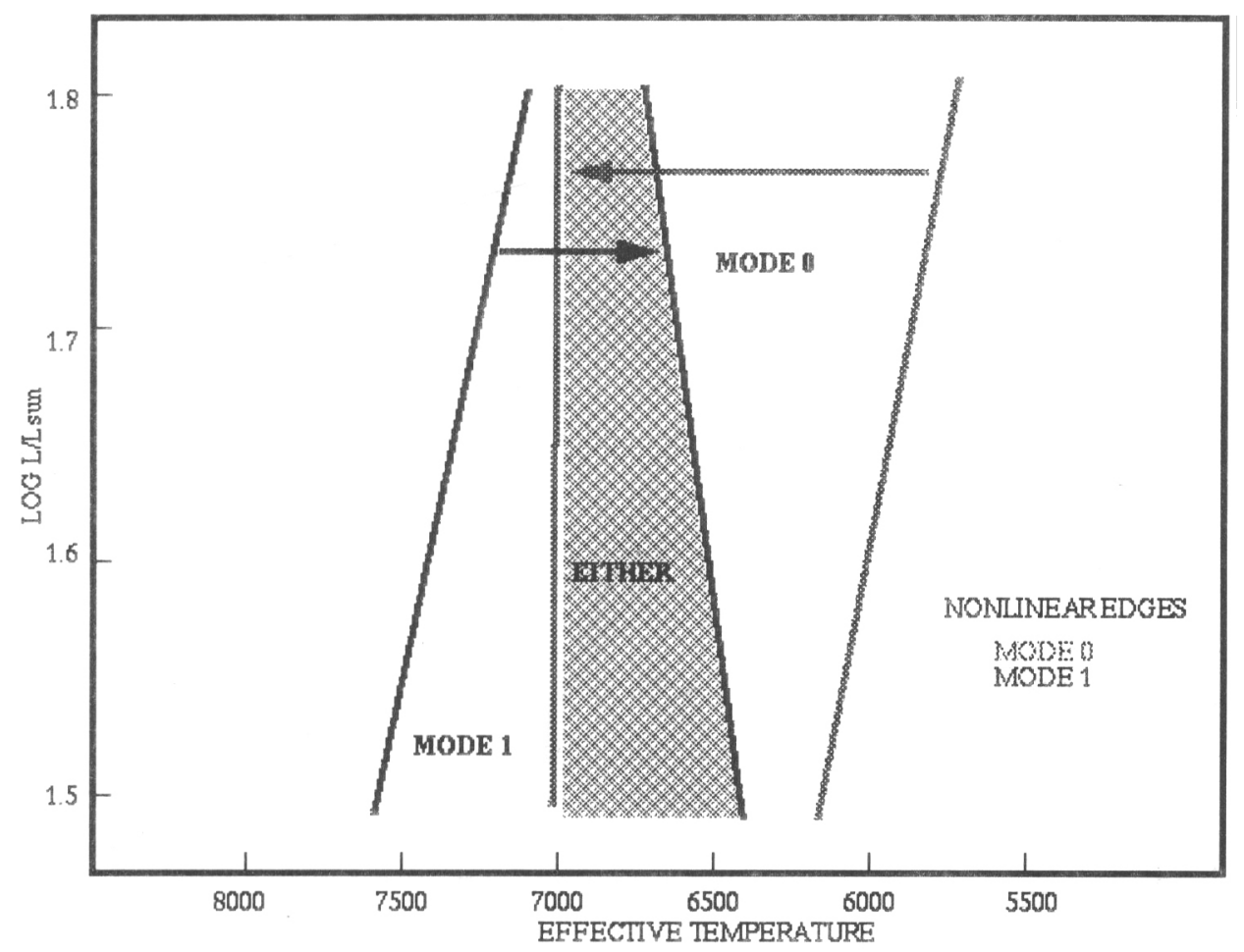

Figure 3: Areas of nonlinear mode preference in the HR diagram.

The region between the two shaded areas in Figure 2 is the "hysteresis region" in which either mode is a possible full amplitude candidate (also called the "either-or" region). This region is shown as a shaded area in Figure 3, above, which also emphasizes the overlapping instability strips for the two modes studied.

Stars evolving to the red will first encounter instability at the overtone blue edge. Overtone pulsations will be stable limit cycles as the star continues to evolve until the far edge of the "eitheror" region is reached at the overtone nonlinear red edge. At this point they will switch to the fundamental and continue in this mode until stabilized by convection at the fundamental red edge. Similarly, stars evolving toward the blue will begin pulsating in the fundamental mode at the fundamental red edge and continue until the fundamental blue edge is encountered, this time at the blue edge of the "either-or" region. The star then switches to the overtone until stabilized at the overtone blue edge.

Cluster variables will switch modes at one or the other of the switching lines, depending on the direction of evolution. The fraction of overtone pulsators will depend strongly on the evolution direction and weakly on the temperature of the horizontal branch. Dependences on mass and helium abundance are also expected to be weak, and will be addressed in future work. In Figure 3 at $\log \mathrm{L}$ $=1.7$ there should be roughly equal numbers of overtone and fundamental pulsators for redward evolution, but about $25 \%$ overtone pulsators for blueward evolution. This is the sort of difference found between Oosterhoff I and II clusters. Detailed comparisons with specific cluster data are underway. 


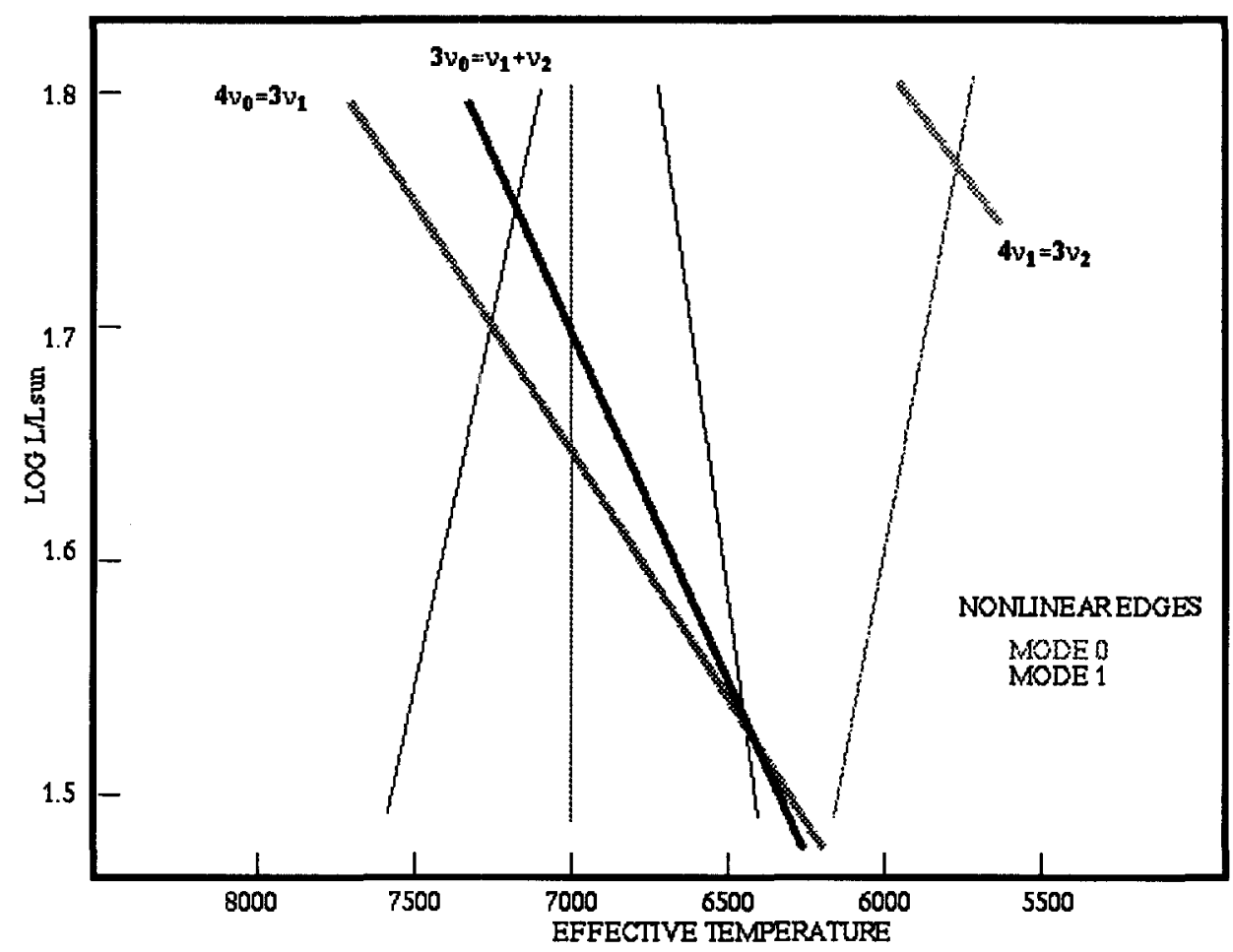

Figure 4: Mode resonance lines in the HR diagram for the current survey.

\section{Mixed-Mode RR Lyrae Stars}

RR Lyrae stars showing a mixture of modes (RRd stars) were discovered in M15 by Cox, Hodson and Clancy (1983), and have now been found in many other clusters as well as the field. See other papers in this collection for the current observational status of these objects. Although several models have been constructed showing this behavior, the observed location of the stars in a narrow range of temperatures near the center of the instability strip has not been reproduced theoretically. Possible explanations of these stars include 1) mode switching at a transition line (in Figure 4, the lines at $6500 \mathrm{~K}$ and $7000 \mathrm{~K}$ ), 2) a stable "mixed-mode" region in the HR diagram instead of the "either-or" region found here, and 3) mixed-mode behavior near resonances (see Buchler and Kovacs, 1986). Some likely resonance lines are shown in Figure 4 for the current survey. Linear combinations of these lines are also possible, but should lie in the same general vicinity as the lines shown.

Some preliminary computations have been undertaken with the present models to determine the credibility of these explanations. Only a few models have been computed, so the conclusions are tentative. The computations take the form of time integrations starting with either a relatively pure mode at low amplitude, or a mixture of modes, and continuing until the final nonlinear result can be ascertained. During the calculation, the amplitudes of the components are obtained using the PDM technique (Stellingwerf, 1978). Two models will be discussed here, one at relatively high luminosity (model 2.3), and one at low luminosity (model 4.6). 


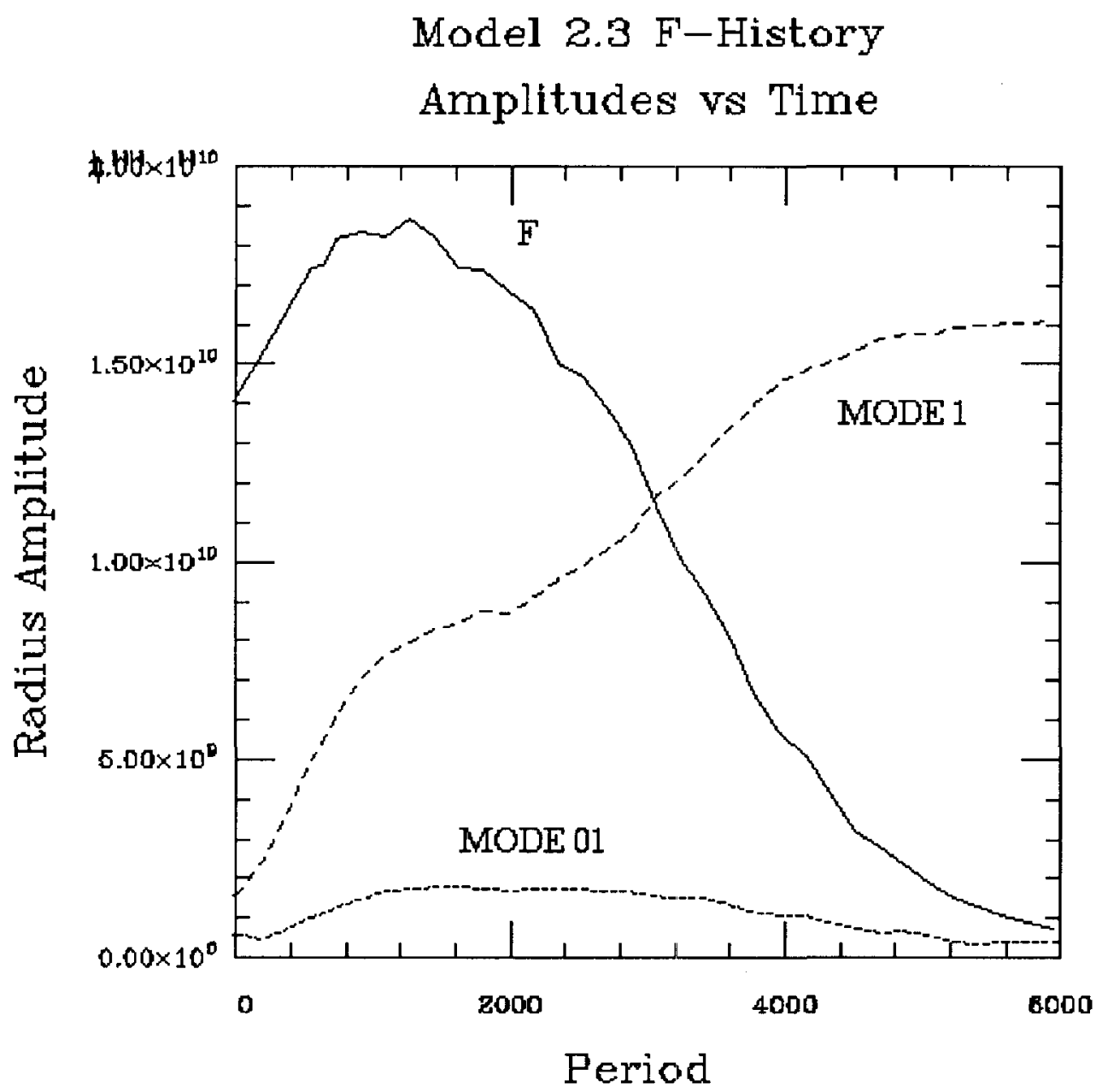

Figure 5: Time history of the radius amplitudes of the fundamental, overtone, and the nonlinear mode coupling term in the spectral analysis of model 2.3 .

Model 2.3 has $\log \mathrm{L}=1.8, \mathrm{~T}_{\mathrm{e}}=7100 \mathrm{~K}$. Figure 5 shows the evolution of this model when initiated in the fundamental mode. At first both the fundamental and the first overtone grew, until about period 1000 , when it appeared that a stable mixture of modes was emerging. Unfortunately, this was not the case since the overtone eventually dominated and quenched the larger amplitude fundamental. Note that the switch took 6000 periods, or about 9 years. Since this model is not exactly at the transition line, an actual mode switch at the line will be much longer (perhaps 100-1000 years, depending on the rate of evolution). Models at lower $\mathrm{L}$ will take longer still because of smaller growth rates. These switching times are much longer than those found for purely radiative models. It thus appears that mode switching is a possibility to be considered, and should be checked as the observational time base is extended. 


\section{Model 4.6/1 - History \\ Amplitudes vs Time}

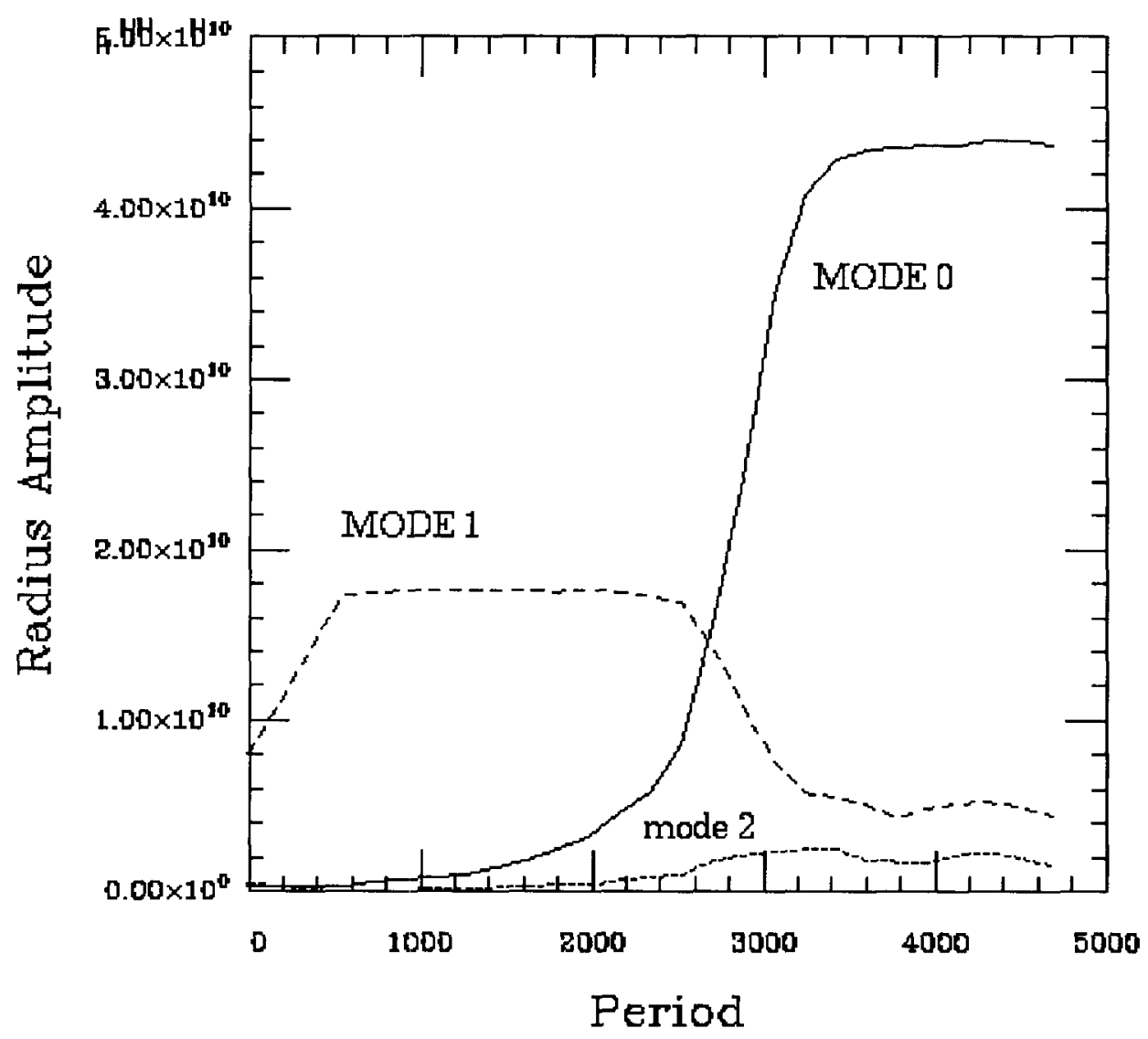

Figure 6: Time history of the first three modes in the development of model 4.6 initiated in the overtone.

Model 4.6 has $\log \mathrm{L}=1.5$ and $\mathrm{T}_{\mathrm{e}}=6500 \mathrm{~K}$. This model is thus near the two main resonance lines in Figure 4, as well as the cool mode switching line. Also, its linear growth rates are smaller than model 4.6. Figure 6 shows the evolution of the model when initiated in the overtone mode. After 500 periods of integration the overtone establishes itself at a stable limit cycle, and maintains this behavior until period 2500 . The light and velocity curves look perfectly regular all during this period, and the model would certainly be classified as a stable overtone with the usual visual analysis. The PDM amplitudes, however, show that the fundamental begins to grow at about period 1000 , and is dominant by period 3000 . The overtone decays, but does not disappear, and the second overtone also joins in toward the end. The two overtones continue at about a $10 \%$ level in radius amplitude, which is about $30 \%$ in luminosity. A long term oscillation is also visible, with a period of about 1000 periods (about 1 year). 


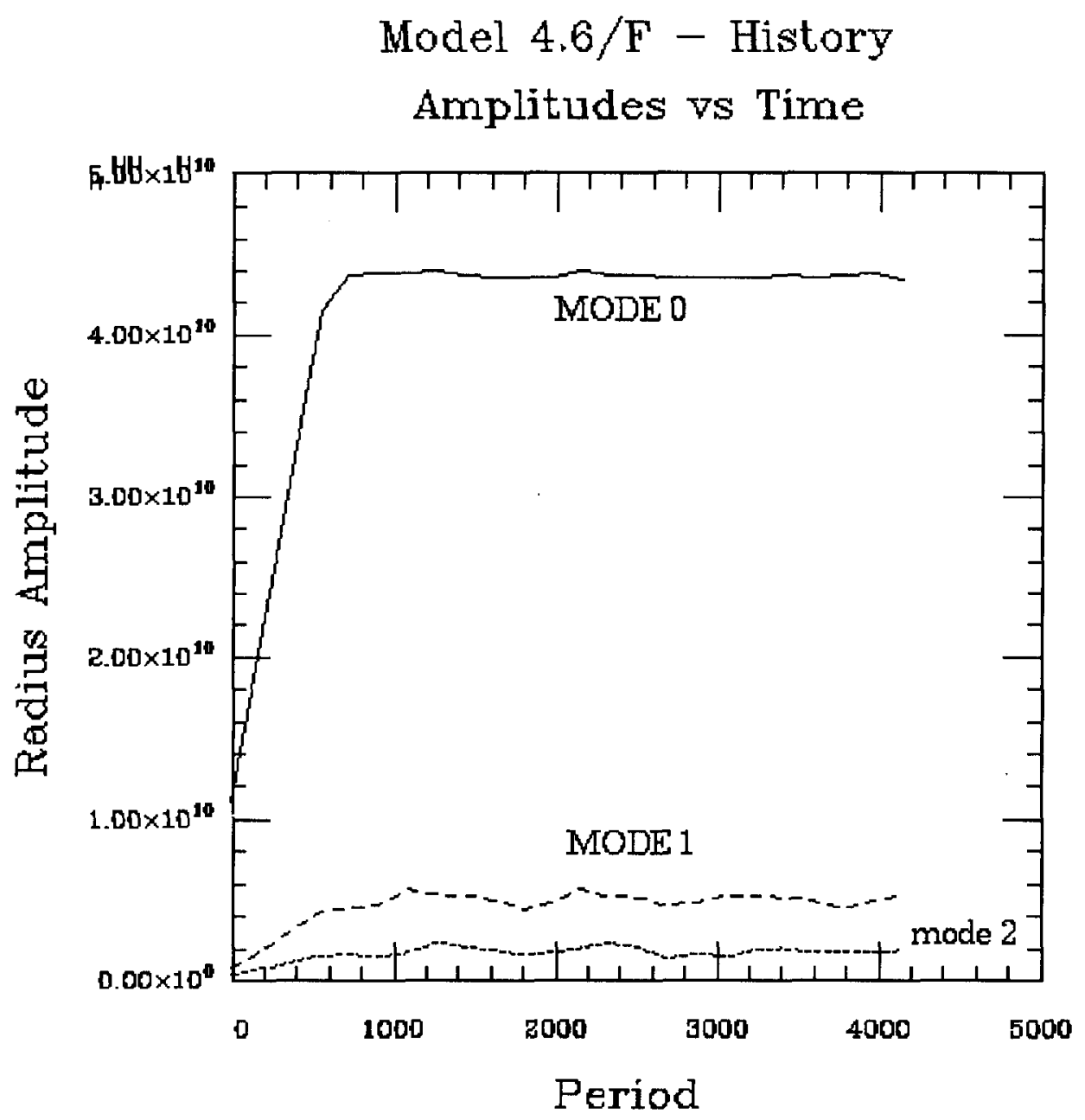

Figure 7: Time history of the first three modes in the development of model 4.6 initiated in the fundamental.

To test whether the long term behavior seen in Figure 6 is really a stable mixed-mode limit cycle, the same model was initialized in the fundamental mode and another long integration undertaken. The result is shown in Figure 7. In this case the model proceeds directly to the same behavior seen after 3500 periods in Figure 6 , and is at the final state after about 500 periods. Again the long term modulation is seen in the secondary modes, and both appear to be in phase with about a 1000 period modulation time. This behavior is suggestive of a three mode resonance, but it is not clear whether this phenomenon is identical to that seen in actual RRd stars, or if the resonance actually plays a vital role. In particular, a model at this same luminosity, but at a temperature of $7000 \mathrm{~K}$ (near the other transition line) also appears to show signs of a mixed mode state, but this time with the overtone dominant. Further tests will be needed to finally resolve these issues. 


\section{Conclusion}

The convective RR Lyrae survey has supplied a new prediction for the instability strip that differs in several significant features from previous surveys. These differences still need to be carefully compared with observational evidence, but indications are that better agreement will be achieved for the RR Lyrae systematics. Amplitudes of the models are generally lower than radiative models, and the growth rates are smaller. This should favor mixed mode behavior, but preliminary results seem to indicate that this is true only at low luminosities. We find that the time required to switch modes is longer than previously derived, however, opening the possibility that the RRd stars are in transit from one mode to another. If this is true, then changes should be seen in the amplitudes of at least some of these objects as a function of time.

\section{References:}

Baker, N. H., and Kippenhahn, R., 1962, Zeit. f. Ap. 54, 114.

Baker, N. H., and Gough, D., 1979, Ap. J. 234, 232.

Buchler, J. R., Kovacs, G., 1986, Ap. J. 303, 749.

Christy, R. F., 1966, Ap.J. 145, 337.

Cox, A. N., Brownlee, R. R., and Eilers, D. D.,1966, Ap. J. 144, 1024.

Cox, A. N., Hodson, S. J., and Clancy, S. P., 1983, Ap. J. $266,94$.

Deupree, R. G., 1979, Ap.J. 234, 228.

Stellingwerf, R. F., 1978, Ap.J. 224, 953.

Stellingwerf, R. F., 1982, Ap.J. 262, 330.

Unno, W., 1967, PASJ 19, 140.

Wood, P. R., 1979, Ap.J. 227, 220.

\section{Acknowledgments:}

This work has been supported in part by the National Science Foundation, the Department of Energy, and Trieste Observatory.

\section{Questions:}

S. R. Sreenivasan: The system of equations you have used is of the "reaction-diffusion" type of equations. They exhibit structural phase transitions, hystersis, and transition to chaos. There is considerable literature on the subject and it might be interesting to make comparisons with your work.

RFS: I agree!

Geza Kovacs: Is not the eddy viscosity just another fudge factor to substitute the artificial viscosity by a "more physical one"?

RFS: We find that the amplitude is only weakly dependent on the eddy viscosity. Remember that the turbulent pressure is one component in the total pressure, and that it is the total pressure that is fixed by the momentum equation. In one test, I turned off the turbulent viscosity completely, but the total work was affected only slightly, although the eddy dissipation was significant. The structure had adjusted to maintain nearly the same total pressure. The eddy term will have a secondary effect on the structure, which is weaker than it looks. In most of our models, limiting amplitude is determined by strong convective quenching, primarily at the phase near minimum radius, rather than by viscous effects. 